\title{
LA CONJUGAISON DU VERBE FRANÇAIS BASÉE SUR LE CODE ORAL
}

\author{
Martin Pleško
}

\begin{abstract}
This paper is a comparison of two conjugational systems of the French verbs. The first one, so called traditional conjugation, is based on the infinitive ending of a verb and the second system is based on the phonetic bases of each verb in the Simple Present Tense.

Keywords: verb; conjugation; phonetic base; infinitive.

Résumé : Cet article compare deux types de conjugaison des verbes français : la conjugaison dite traditionnelle basée sur la terminaison du verbe à l'infinitif et la conjugaison par bases phonétiques qui regroupe les verbes selon leur nombre de bases orales au présent de l'indicatif.

Mots-clés : verbe ; conjugaison ; base phonétique ; infinitif.
\end{abstract}

\section{Introduction}

Les échanges sociaux et langagiers sont pratiquement impossibles sans verbes. Ceux-ci sont essentiels si l'on souhaite communiquer. Depuis plusieurs années, l'approche communicative et l'approche actionnelle prévalent en classe de langues étrangères (FLE). Cuq et Gruca (2002 : 245) expliquent que l'approche communicative vise le développement de la compétence communicative qui ne se limite pas aux règles grammaticales mais implique aussi la connaissance des règles socioculturelles de l'emploi de la langue, la cohérence et la cohésion et les compétences stratégiques de la communication. L'approche actionnelle est encore plus moderne puisqu' elle considère tous les utilisateurs/ apprenants de la langue comme des acteurs sociaux ayant à accomplir des tâches dans des circonstances données. Ces tâches mobilisent des compétences stratégiques en vue de parvenir à un résultat déterminé (CECR 2001 : 15). Ces deux méthodologies obligent les enseignants de FLE à enseigner de manière efficace la conjugaison des verbes car être capable de conjuguer sans hésitation facilite la communication.

En raisonnant à partir de notre propre expérience dans l'enseignement du FLE, nous soutiendrons le fait que les apprenants sont, en général, amenés à apprendre la conjugaison par cœur, notamment les verbes irréguliers. Cette façon d'apprendre est considérée comme efficace puisque beaucoup de gens, qui l'ont appliquée, maîtrisent le français.

\footnotetext{
1 Cadre européen commun de référence pour les langues.
} 
Néanmoins, cette méthode dite traditionnelle, basée sur la terminaison du verbe à l'infinitif peut poser des problèmes aux apprenants qui sont non réceptifs à l'apprentissage par cœur. Cette conjugaison, que le français a conservé du latin, ne représente pas la seule voie à suivre dans l'enseignement. L'objectif de cet article est de présenter la conjugaison non traditionnelle, basée sur le classement phonétique des verbes.

D'un côté, la conjugaison non traditionnelle n'oblige pas à apprendre par cœur, mais elle permet de dériver des formes. Si nous connaissons la/les base(s) phonétique(s) d'un verbe au présent de l'indicatif, nous serons capables de le conjuguer à n'importe quel temps et mode. De l'autre côté, ce type de conjugaison est lié au code oral de la langue française, ce qui nécessite la connaissance d'une transcritpion phonétique, par ex. : alphabet phonétique international (API). À partir de critères historiques, les verbes sont regroupés selon leur terminaison à l'infinitif plus les verbes irréguliers et le classement traditionnel repose essentiellement sur l'analyse graphique (Dubois 1967 : 56). Ce classement est aussi appliqué lors des cours de français en tant que langue maternelle. Pourtant, le classement phonétique nous paraît meilleur puisqu'il permet d'éviter un apprentissage par cœur systématique.

\section{Conjugaison selon la terminaison de l'infinitif : classement traditionnel}

Les verbes sont traditionnellement classés selon leur flexion à l'écrit. Le classement traditionnel reconnaît trois groupes de verbes dont chacun a ses particularités. Riegel, Pellat et Rioul (2008 : 264) s'opposent à ce classement et affirment qu'il ne suffit pas de connaître l'infinitif d'un verbe, ni sa base écrite pour pouvoir le conjuguer. De leur côté, Ducháček et Bartoš (1974 : 133) expriment l'opinion qu'il suffit de connaître seulement l'infinitif, le présent de l'indicatif, le passé simple et le participe passé pour pouvoir construire toutes les formes d'un verbe. Ce n'est toutefois pas le cas de tous les verbes irréguliers.

L'élément fondamental pour conjuguer un verbe est son radical qui indique la signification du verbe, son idée générale et qui est identique dans tous les mots de la même famille. Aux radicaux s'ajoutent, dans le cas des verbes, d'autres éléments variables pour produire les formes d'un paradigme. Ce ne sont que des formes graphiques qui sont prises en considération et toute variation graphique, par exemple : peler, détermine éventuellement l'existence d'un sous-groupe (Dubois $1967: 57$ ).

Voici quelques brèves caractéristiques des trois groupes de verbes :

a) Les verbes en -er représentent environ $90 \%$ des verbes français. À quelques exceptions près (certaines particularités orthographiques comme peler et achever, lever, mener, peser, etc.), ils sont tous réguliers. En raison de l'ajout régulier de nouveaux verbes, notamment de ceux rendus nécessaires par l'évolution de l'activité humaine, leur liste est ouverte. Les verbes de ce type ont un radical unique au présent de l'indicatif (Riegel Pellat - Rioul 2008 : 272).

b) Les verbes en -ir ont un radical différent au singulier, au pluriel et au participe présent. Ce groupe est moins étendu, il n'est constitué que d'environ 300 verbes. La productivité est zéro, sauf les verbes alunir et aterrir.

c) Les irréguliers en -ir, -oir, -re, etc. et les verbes avoir, aller, être et faire. Les onze verbes les plus employés en français font partie de ce groupe : avoir, être, faire, dire, aller, voir, savoir, pouvoir, falloir, vouloir, venir. 
La répartition en trois groupes est déséquilibrée puisque les verbes en -er sont très nombreux et moins utilisés alors que les verbes du dernier groupe sont en moindre quantité mais fréquemment employés (Riegel - Pellat - Rioul 2008 : 264).

\section{Conjugaison par bases phonétiques : classement phonétique}

Il existe trois catégories qui correspondent au nombre de bases orales qu'un verbe peut avoir au présent de l'indicatif, plus quatre verbes irréguliers (avoir, aller, être, faire). Abry et Chalaron $(2000: 6)$ proposent un classement complet des verbes français suivant le nombre de leurs bases orales :

\begin{tabular}{|c|c|c|c|c|}
\hline \multirow{2}{*}{ Verbes à 1 base } & \multicolumn{2}{|c|}{ Verbes à 2 bases } & \multirow{2}{*}{ Verbes à 3 bases } & \multirow{2}{*}{ Verbes irréguliers } \\
\hline & Type 1 & Type 2 & & \\
\hline \multirow[t]{2}{*}{$1 \mathrm{je}$} & $1 \mathrm{je}$ & 1 je & $1 \mathrm{je}$ & \multirow{4}{*}{$\begin{array}{l}\text { aller } \\
\text { avoir } \\
\text { être }\end{array}$} \\
\hline & 2 tu & $2 \mathrm{tu}$ & 2 tu & \\
\hline \multirow{2}{*}{4 nous } & 3 il/elle & 3 il/elle & 3 il/elle & \\
\hline & 6 ils/elles & 4 nous & 4 nous & \\
\hline 5 vous & 4 nous & 5 vous & 5 vous & \multirow[t]{2}{*}{ faire } \\
\hline 6 ils/elles & 5 vous & 6 ils/elles & 6 ils/elles & \\
\hline
\end{tabular}

À l'exception des verbes irréguliers, tous les verbes français possèdent la même forme orale au singulier (personnes 1, 2, 3).

Les traits caractéristiques de chaque catégorie:

a) Les verbes à une base ont la même base phonétique à toutes les personnes. Hormis quelques exceptions (acheter, appeler, jeter, céder, répéter, noyer, appuyer, essayer, etc.), tous les verbes se terminant en -er et quelques verbes du troisième groupe (par exemple : offrir, souffrir, ouvrir, couvrir, cueillir, assaillir, défaillir, tressaillir, courir, secourir, rire, sourire, conclure, exclure, inclure, etc.) appartiennent à cette catégorie selon le classement traditionnel.

b) La catégorie des verbes à deux bases se subdivise en deux types en raison des changements orthographiques au pluriel (personnes 4, 5,6) :

- type 1 : une même base pour les personnes 1,2,3,6 et une autre pour les personnes 4,5 ,

- type 2 : une base pour les personnes 1, 2, 3 et une autre pour les personnes 4, 5, 6 .

Le principe de conjugaison des verbes à deux bases est l'alternance de la base courte et de la base longue. Le type 1 comprend des verbes comme acheter, appeler, jeter, céder, répéter, noyer, appuyer, essayer, etc. et quelques verbes irréguliers selon le classement traditionnel, par exemple : croire, voir, extraire, fuir, mourir, acquérir, s'asseoir. Le type 2 englobe les verbes en -ir et la majorité des verbes irréguliers en -aître, -oître, -être, -aindre, -oindre, -eindre, -ordre, -endre (prendre et ses composés sont exclus) selon le classement traditionnel et les verbes savoir, valoir, résoudre. 
c) Les verbes à trois bases sont surtout, selon le classement traditionnel, les verbes irréguliers et leurs composés. Ils ont une même base pour les personnes 1, 2, 3, une autre pour les personnes 4,5 et une troisième pour la personne 6 . Cette catégorie ne contient pas beaucoup de verbes, par exemple : pouvoir, vouloir, devoir, boire, recevoir, percevoir, tenir, venir et prendre.

d) Les verbes irréguliers doivent être appris par cœur : aller, avoir, être, faire.

La conjugaison par bases orales peut paraître plus compliquée puisque, comme il est mentionné ci-dessus, elle exige la connaissance d'une transcription phonétique. Cependant, cette méthode peut être perçue comme étant plus productive du point de vue pratique.

\section{Verbes modèles}

Dans le tableau suivant, à l'aide des verbes modèles courir (1 base), voir (2 bases, type $1)$, finir (2 bases, type 2 ) et boire (3 bases), on présente la conjugaison sur le code oral du verbe :

\begin{tabular}{|c|c|c|c|c|c|}
\hline \multirow[b]{2}{*}{ Pronoms sujets } & \multicolumn{4}{|c|}{ Base(s) } & \multirow{2}{*}{$\begin{array}{c}\text { Suffixes } \\
\text { X Tps Pers }\end{array}$} \\
\hline & $\begin{array}{c}1 \\
\text { courir }\end{array}$ & $\begin{array}{l}2 \text { (type 1) } \\
\text { voir }\end{array}$ & $\begin{array}{l}2 \text { (type 2) } \\
\text { finir }\end{array}$ & $\begin{array}{c}3 \\
\text { boire }\end{array}$ & \\
\hline 32 & kuR & vwa & fini & bwa & $\varnothing \varnothing \varnothing$ \\
\hline ty & kuR & vwa & fini & bwa & $\varnothing \varnothing \varnothing$ \\
\hline il, $\varepsilon l, \tilde{o}$ & kuR & vwa & fini & bwa & $\varnothing \varnothing \varnothing$ \\
\hline $\mathrm{nu}(\mathrm{z})$ & kuR & vwaj & finis & byv & $\varnothing \varnothing \widetilde{\text { o }}$ \\
\hline $\mathrm{vu}(\mathrm{z})$ & kuR & vwaj & finis & byv & $\varnothing \varnothing$ е \\
\hline il(z), $\mathrm{cl}(\mathrm{z})$ & kuR & vwa & finis & bwav & $\varnothing \varnothing \varnothing$ \\
\hline
\end{tabular}

(1) Le pronom sujet indique le genre et le nombre. (2) $X$ = suffixe supplémentaire. En général, il s'agit de $-R[\varnothing]$ (présent et imparfait de l'indicatif) ou $+R[R]$ (futur simple, conditionnel présent). (3) Tps = suffixe de temps. (4) Pers = suffixe de personne qui ne varie jamais, il est discontinu. (5) $\varnothing=$ suffixe zéro.

La conjugaison des verbes français suivant leur classement phonétique ne consiste qu'à l'ajout simple d'un suffixe à la base orale (Pavlík 1991 : 20-46). Différentes séquences phonologiques sont allomorphes si la substitution d'une séquence à une autre n'est pas distincte, et si, par voie de conséquence, des segments différents dénotent une même valeur sémiologique, par exemple $: \hat{e}$ tre $\rightarrow$ je suis [zəsui], tu es [tyc] ; les séquences [sui] et [ع] sont les allomorphes (Camussi-Ni 2006). 


\section{Conclusion}

La conjugaison des verbes français est une question complexe. Les Français conjuguent les verbes mécaniquement. Pour un étranger, cela n'est pas une acquisition automatique mais découle de procédés de mémorisation. Ce n'est qu'après un certain temps d'apprentissage que cela devient automatique bien qu'il reste toujours des lacunes. Jusqu'à aujourd'hui, les enseignants apprennent à conjuguer selon le classement traditionnel non seulement en cours de FLE hors du territoire français/ francophone, mais également dans les établissements d'enseignement français. Selon Marie-Armelle Camussi-Ni (2006), les trois groupes de verbes n'ont plus de valeur. Le classement phonétique est plus détaillé et il ne reconnaît que quatre verbes irréguliers. Le tableau suivant récapitule des avantages et des inconvénients du classement traditionnel et du classement phonétique :

\begin{tabular}{|c|c|c|c|}
\hline \multicolumn{2}{|c|}{ Classement traditionnel } & \multicolumn{2}{|c|}{ Classement phonétique } \\
\hline+ & - & + & - \\
\hline $\begin{array}{l}\text { Fonctionne depuis } \\
\text { longtemps }\end{array}$ & \begin{tabular}{|l|} 
Les trois groupes \\
verbaux n'ont plus \\
de valeur
\end{tabular} & $\begin{array}{l}\text { Il est possible de } \\
\text { dériver certaines } \\
\text { formes }\end{array}$ & $\begin{array}{l}\text { Connaissance de } \\
\text { l'API nécessaire }\end{array}$ \\
\hline $\begin{array}{l}\text { Idéal pour les jeunes } \\
\text { élèves et au début de } \\
\text { l'apprentissage }\end{array}$ & $\begin{array}{l}\text { Apprentissage par } \\
\text { cœur systématique, } \\
\text { trop de verbes } \\
\text { irréguliers }\end{array}$ & $\begin{array}{l}\text { Moins } \\
\text { d'apprentissage par } \\
\text { cœur }\end{array}$ & $\begin{array}{l}\text { Système plus } \\
\text { complexe: } \\
\text { apprentissage plus } \\
\text { difficile au début } \\
\end{array}$ \\
\hline \multirow[t]{2}{*}{$\begin{array}{l}\text { Classement respecté } \\
\text { dans les manuels de } \\
\text { FLE }\end{array}$} & $\begin{array}{l}\text { Beaucoup } \\
\text { d'analogies erronées }\end{array}$ & $\begin{array}{l}\text { Seulement } 4 \text { verbes } \\
\text { irréguliers }\end{array}$ & $\begin{array}{l}\text { Les manuels de FLE } \\
\text { n'utilisent pas ce } \\
\text { classement }\end{array}$ \\
\hline & & $\begin{array}{l}\text { Idéal pour les } \\
\text { apprenants en } \\
\text { philologie et pour } \\
\text { les élèves des lycées } \\
\text { bilingues }\end{array}$ & $\begin{array}{l}\text { Certains temps } \\
\text { verbaux demandent } \\
\text { un apprentissage } \\
\text { particulier de la base } \\
\text { orale, par exemple: } \\
\text { voir }\end{array}$ \\
\hline
\end{tabular}

En combinant la conjugaison traditionnelle et la conjugaison par bases phonétiques, nous pourrions aboutir à une conjugaison parfaite. À l'aide du classement traditionnel, il serait plus facile d'apprendre le classement phonétique et celui-ci permettrait d'éliminer le nombre de verbes irréguliers et donc l'apprentissage par cœur. L'enseignant reste cependant le seul maître de la décision d'utiliser cette méthode.

\section{Bibliographie}

Aвry, Dominique - Chalaron, Marie-Laure (2000), La grammaire des premiers temps - volume 1, Grenoble : PUG.

Abry, Dominique - Chalaron, Marie-Laure (1999), La grammaire des premiers temps - volume 2, Grenoble : PUG.

Boularès, Michèle - GRAND-Clément, Odile (2000), Conjugaison progressive du français avec 400 exercices, Paris : CLE International.

Cadre européen commun de référence pour les langues : apprendre, enseigner, évaluer (2001), Paris : Conseil de l'Europe. 
Camussi-Ni, Marie-Armelle (2006), Analyse formelle et conceptuelle des formes verbales du français contemporain : À la croisée du passé simple et de l'imparfait, du futur et du conditionnel, les concepts \pm potentiel et \pm défini, Rennes : Université Rennes 2.

Cur, Jean-Pierre - GrucA, Isabelle (2002), Cours de didactique du français langue étrangère et seconde, Grenoble : PUG.

DuboIs, Jean (1967), Grammaire structurale du français : le verbe, Paris : Larousse.

DuCHÁČEK, Otto - BARTOŠ, Jozef (1974), Grammaire du français contemporain, Bratislava : SPN.

Pavlík, Miroslav (1991), Orální morfologie francouzského slovesa, Olomouc : Univerzita Palackého.

Riegel, Martin - Pellat, Jean-Christophe - Rioul, Réné (2008), Grammaire méthodique $d u$ français, Paris : PUF.

Martin Pleško

Katedra romanistiky

Filozofická fakulta

Univerzita Palackého v Olomouci

Křížkovského 8

77180 Olomouc

République tchèque

martin.plesko@gmail.com 